\title{
Anthraquinones from Morinda elliptica
}

\begin{abstract}
Phytochemical studies on roots of Morinda elliptica have resulted in the isolation of a new anthraquinone, 2-formyl-1-hydroxyanthraquinone, and 10 known anthraquinones. The structures of the anthraquinones were established based on spectral studies.
\end{abstract}

Keyword: Morinda elliptica; Rubiaceae; Anthraquinone; 2-formyl-1-hydroxy-anthraquinone 